\title{
Some monophasic self-stimulation loci in the rock squirrel and rat
}

\author{
JAMES E. KING, UNIVERSITY OF ARIZONA
}

Histological data including photographs of all sites were reported for an unusual laboratory species, the rock squirrel (Citellus variegatus) and an uncommon testing procedure, monophasic self-stimulation through bipolar electrodes. Principal findings were that effective self-stimulation sites frequently were near ineffective sites, and that a higher response rate was obtained more frequently for a lateral than for a medial cathode.

Histological data from self-stimulation studies are rarely reported in detail, an omission which has impeded and often prevented useful comparisons of loci between studies. The omission persists despite an abundance of behavioral self-stimulation data, some of which suggest that by now even negative results should be assessed. Thus, Cooper \& Taylor (1967) obtained self-stimulation from thalamic regions which have usually been characterized as ineffective.

In the hope of providing histological material appropriate for interstudy comparisons, data were compiled from several experiments in our laboratory, in which a total of 21 rock squirrels and 10 rats were tested for monophasic self-stimulation.

Method

Descriptions of the testing procedures for S8, S18, S19, R1, R2, R7, and R9 are given by Wetzel \& King (1966), while procedures for S24, S25, S26, S30, S31, S32, S33, S34, S35, S38, S39, and S40 are given by King \& Wetzel (1967). The shaping of the self-stimulation response in Ss who failed to self-stimulate as well as those who did self-stimulate consisted of at least 30-min training periods on each of four days, with .5 msec, $200 \mu \mathrm{A}$ monophasic pulses delivered at 200 per sec for .2 sec. All Ss were tested with both polarities of the monophasic pulses. Rats RL5 and RL6 selfstimulated only after being given more extensive testing. Results

The principal results may be found in Table 1 and Fig. 1. Electrode materials were .01-in. stainless steel or $90 \%$ platinum-10\% iridium wires. Twenty-one of the $31 \mathrm{Ss}$ self-stimulated. It is apparent that ineffective sites were in several cases near effective sites. Indeed, loci for S20, which failed to self-stimulate, and S24, which self-stimulated, did not differ discernibly. (The hole ventral to the arrow for S20 in Fig. 1 is an artifactual tear rather than an extension of the track.) The lack of conspicuous differences in anatomical positions for effective and ineffective sites was unexpected, since stimulation and shaping practices were simllar to those in common use for biphasic pulses. Nevertheless, in order to determine if more
MARY C. WETZEL, SOUTHERN ARIZONA MENTAL HEALTH CENTER LOUISE E. NOWICKI, SOUTHERN ARIZONA MENTAL HEALTH CENTER extreme conditions might yield self-stimulation, additional testing was given RL5 and RL6. Neither animal had responded during the standard four-day shaping period. For RL6 intensity and train duration were increased on Day 5, and on shaping Day 8 it began responding to a $1-\mathrm{sec}$ train of $.5 \mathrm{msec}$ pulses delivered at 200 per sec, at an intensity of approxdmately

Table 1 Electrode, performance, and localization data

\begin{tabular}{|c|c|c|c|c|}
\hline Subject & Material & $\begin{array}{c}\text { Self- } \\
\text { stimulation }\end{array}$ & $\begin{array}{l}\text { Cathode position } \\
\text { for higher rate }\end{array}$ & Locus \\
\hline R1 & steel & yes & no difference & TMT, ZI \\
\hline $\mathbf{R} 2$ & steel & yes & anterior & F, LH-MFB \\
\hline R6 & steel & no & & $\begin{array}{l}\text { out of brain, } \\
\text { medial to } \mathrm{CP}\end{array}$ \\
\hline R7 & steel & yes & lateral & LH-MFB \\
\hline R9 & steel & yes & lateral & $\begin{array}{l}\text { almost out of brain, } \\
\text { CP }\end{array}$ \\
\hline $\mathbf{R} 10$ & plat. & no & & $\begin{array}{l}\text { almost out of brain, } \\
\text { CP }\end{array}$ \\
\hline $\mathbf{R} 11$ & plat. & no & & $\begin{array}{c}\text { almost out of brain, } \\
\text { LH-MFB }\end{array}$ \\
\hline $\mathbf{R} 12$ & plat. & no & & $\begin{array}{c}\text { almost out of brain, } \\
\text { LH-MFB, CP }\end{array}$ \\
\hline RL5 & plat. & yes & no difference & $\begin{array}{c}\text { almost out of brain, } \\
\text { CP }\end{array}$ \\
\hline RL6 & plat. & yes & lateral & LH-MFB, CP \\
\hline S8 & steel & yes & lateral & ZI \\
\hline S18 & steel & yes & no difference & CP, LH-MFB \\
\hline S19 & steel & yes & posterior-medial & MFB, OC \\
\hline $\mathbf{S} 20$ & steel & no & & $\mathrm{CP}, \mathrm{LH}$ \\
\hline $\mathbf{S 2 3}$ & steel & no & & STh \\
\hline S24 & plat. & yes & lateral & CP, LH \\
\hline$\$ 25$ & plat. & yes & lateral & VPM, GM \\
\hline S26 & plat. & yes & lateral & ZI, LH-MFB \\
\hline $\mathbf{\$ 2 7}$ & plat. & no & & CP, STh \\
\hline $\mathrm{S} 28$ & plat. & no & & VPM, GM \\
\hline $\mathbf{S} 29$ & plat. & no & & VPM, LM \\
\hline $\mathbf{S 3 0}$ & plat. & yes & medial & ZI \\
\hline S31 & plat. & yes & lateral & VPM, LM \\
\hline S32 & plat. & yes & no difference & V \\
\hline S33 & plat. & yes & lateral & V, FF \\
\hline S34 & plat. & yes & (lateral) & ZI \\
\hline$\$ 35$ & plat. & yes & (lateral) & $\mathbf{Z I}$ \\
\hline S37 & plat. & no & & $\mathrm{ZI}$ \\
\hline $\mathbf{S} 38$ & plat. & yes & lateral & ZI, MFB-LH \\
\hline S39 & plat. & yes & no difference & LH-MFB, ZI \\
\hline $\mathrm{S40}$ & plat. & yes & lateral & $Z I$ \\
\hline
\end{tabular}

Note-cathode positions for $S 34$ and $S 35$ are in parentheses since rates were only slightly higher for the lateral than for the medial position. Abbreviations used are: $C P=$ cerebral peduncle, $F=$ formix, $F F=$ fields of Forel, GM=medial geniculate, LH=lateral hypothalamus, LM=medial lemniscus, $M F B=$ medial forebrain bundle, $O C=$ optic chiasma, $S T h=s u b$ thalamic nucleus, TMT=mamillothalamic tract, $V=N$. ventralis thalami, $V P M=N$, ventralis posteromedialis thalami, $Z I=z$ ona incerta. 


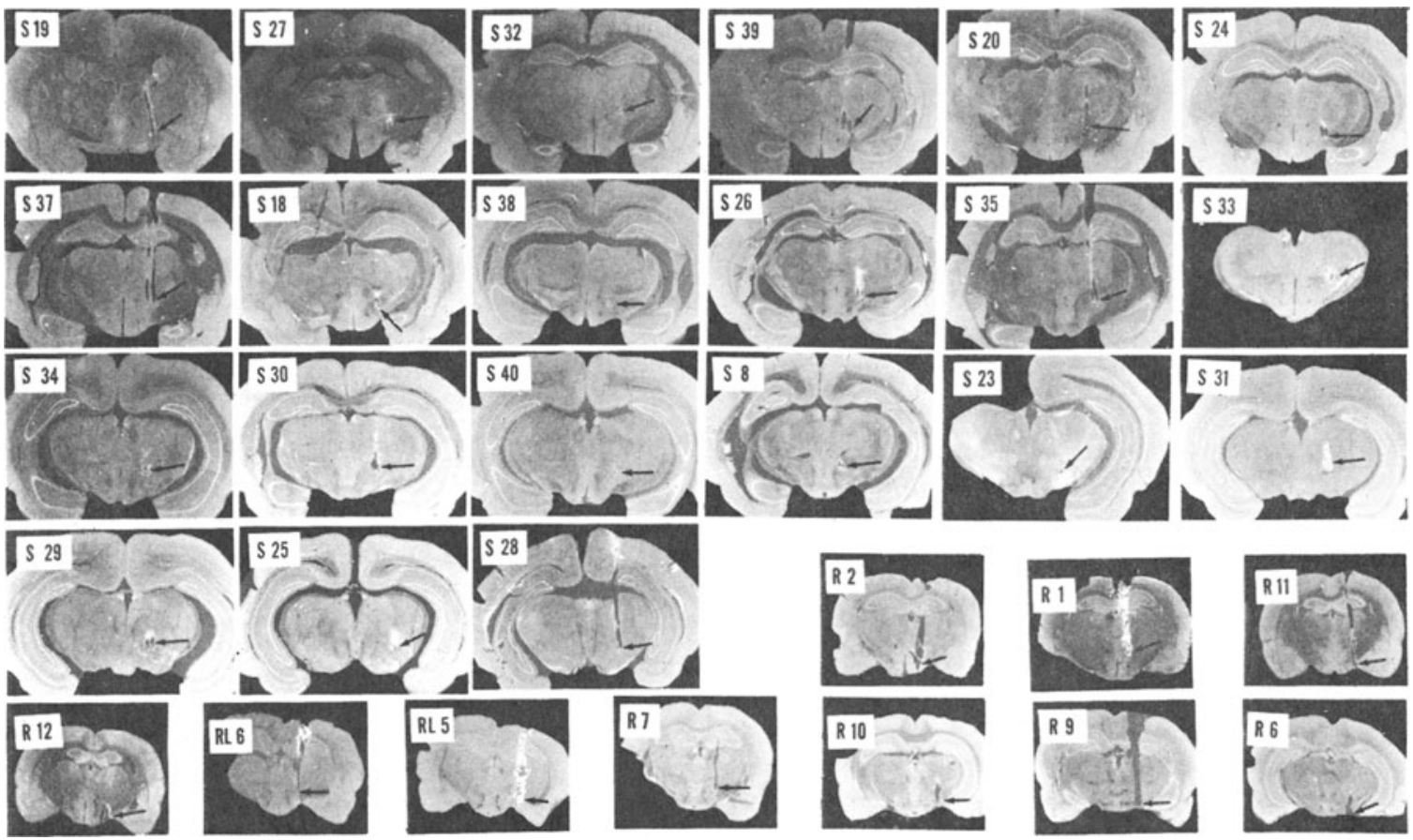

Fig. 1. Photographic negatives of cresyl violet-stained, $40 \mu$ frozen sections. For each species, $S$ (squirrel) and $R$ (rat), sections are ordered from anterior to posterior: from left to right across rows and from top to bottom down rows.

$500 \mu \mathrm{A}$. Self-stimulation was well sustained for eight subsequent days. For RL5 the standard stimulation and shaping procedures were continued beyond the four-day shaping period, and self-stimulation was obtained on shaping Day 7. The electrodes for this animal were found to be, like those of "failures" R10, R11, R12, and self-stimulating R9, almost completely through the base of the brain.

Of the 21 self-stimulating Ss, five failed to respond at different rates for the two polarities. King \& Wetzel (1967) found that seven of eight Ss showing a marked polarity rate difference had responded at a higher rate when the lateral electrode was the cathode. From the other experiments, four more Ss also responded at higher rates for a lateral cathode. The remaining two Ss which yielded a rate difference (R2 and S19) had electrodes which were not oriented in the frontal plane.

\section{Discussion}

The similarity of effective and ineffective sites illustrates the need for more rigorous methods of mapping self-stimulation sites within restricted portions of the brain. An obvious requirement is for more detailed comparisons. The initial failure of RL5 and RL6 to respond would have led to their elimination under conventional testing procedures. That these Ss eventually self-stimulated suggests the need for extensive testing of $\mathrm{Ss}$ that fail initial self-stimulation criteria. Sites may be compared meaningfully between studies only if such behavioral detalls are available.
This is particularly essential for such an atypical site as that of RL5, whose electrode was almost out of the brain, and for whom the region of effective electrical stimulation included only a small portion of the cerebral peduncle.

Data are still too few to warrant hypotheses as to why a lateral cathode usually yielded a higher selfstimulation rate than a medial cathode. In the present experiments only two cathode sites were tested per $S$, and the existence of a gradient of stimulation effectiveness from medial to lateral, with lateral cathodes most effective (King \& Wetzel, 1967), would have to be verified by other means. One such method might be to determine polarity effects for a number of test sites within one frontal plane for individual Ss. That the relationship may not be simple is suggested by the fact that gross loci for S30 and S40 were almost identical; yet response rate was higher for a medial cathode for $\$ 30$ and a lateral cathode for $\$ 40$. Further, there are no data yet concerning polarity effects for electrodes oriented in longitudinal and other planes.

\section{References}

COOPER, R. M., \& TAYLOR, L. H. Thalamic reticular system and central grey: self-stimulation. Science, 1967, 156, 102-103.

KING, J. E., \& WETZEL, MARY C. Self-stimulation in the rock squirrel as a function of current direction. Psychon. Sci, 1967, in press. WETZEL, MARY C., \& KING, J. E. Self-stimulation with monophasic current in the rock squirtel and rat. Psychon. Sci, 1966, 6, 7-8. 